\title{
Invasive aspergillosis in a case of suspected lung
}

\section{mass}

\begin{abstract}
We present a distinct and characteristic case report of disseminated angioinvasive aspergillosis. A middle-aged female presented with shortness of breath and coughing up of blood. She was taken to a hospital. The decedent's condition was declining at the emergency room. X-ray chest and emergent CT scan demonstrated a lung mass. The differential diagnoses included malignancy, sarcoidosis and infectious disease. Aspergillosis was at the lower end of the differential diagnosis. No extensive tests were performed as a diagnostic work-up since the decedent's condition was deteriorating rapidly. She underwent a lung lobe resection for a suspected lung mass with recurrent hemoptysis. After lung lobe resection, her condition continued to decline and she died at the hospital within two weeks following surgery. Histopathologic examination of lung and brain tissues revealed necrotizing tissues with hemorrhagic borders in lungs; multiple cavitating, hemorrhagic, necrotic, soft yellow abscesses in lungs, cerebrum, and cerebellum. Gomori Methenamine Silver (GMS) staining showed septate hyphae branching dichotomously at acute angles, consistent with Aspergillus fungus. A case is presented here who expired from disseminated angioinvasive aspergillosis involving lung and brain, where provisional diagnosis was a lung mass of unknown etiology.
\end{abstract}

Keywords: aspergillosis, septate hyphae, angioinvasive, gomori methenamine silver
Volume 6 Issue I - 2018

\author{
Altaf Hossain, Emran Altaf, Renae Berry, Erik \\ K Mitchell \\ Forensic Medical of Kansas City, USA
}

Correspondence: Altaf Hossain, 40S I8th Street, Kansas city, Kansas 66102, USA, Tel 913-299-1533, Fax 913-912-1388, Email ahos191276@aol.com

Received: November 20, 2017 | Published: January 17, 2018
Abbreviations: GMS, gomori methenamine silver; COPD, chronic obstructive lung disease

\section{Introduction}

Aspergillosis is caused by the fungus Aspergillus, which is commonly found in nature but only rarely causes illness in people. The aspergilli may cause an invasive disease in immunocompromised patients and in patients with hematological malignancies, hematopoietic stem cell or solid organ transplants, congenital or acquired immunodeficiency, as well as use of corticosteroids and other immunosuppressive drugs. Aspergillosis is caused by Aspergillus fumigatus, flavus and less commonly by A terreus, nidulans and niger. Aspergillus fumigatus is the most prevalent fungal pathogen responsible for fatal invasive aspergillosis. The most common portal of entry is the respiratory system. ${ }^{1}$ Invasive pulmonary aspergillosis is the most serious type of aspergillosis infection. It can affect any organ, particularly the heart, lungs, brain, and kidneys. The central nervous system is one of the most frequent involvements of invasive aspergillosis besides the lungs. The infections mostly occur through hematogenous dissemination from a focus, such as lung infection. We report here a case where the decedent died of disseminated angioinvasive aspergillosis involving lung and brain, where initial diagnosis was a lung mass of unknown etiology.

\section{Case}

A 49-year-old female had a history of shortness of breath, pneumonia, chronic obstructive lung disease (COPD), and chronic soft tissue infection in her right arm. The medical record review indicates clinically diagnosed repeated fungal infection in right arm of unknown etiology. Combination of itraconazole and terbinafine was given and the cutaneous infection of the arm was healed. She had coughed up blood, so she was taken to hospital where she had lung lobe resection for suspected lung mass. The decedent's condition was declining at the emergency room. X-ray chest and emergent CT scan showed a lung mass. The diagnostic and management protocol on a suspected (larger than $8 \mathrm{~mm}$ ) lung mass after CT scan includes, biopsy and/or surgical resection. The working diagnosis was an unknown lung mass. It was decided to do a lung resection, because the decedent was coughing up blood. The differential diagnoses included lung cancer, lymphoma, sarcoidosis, tuberculosis, pneumonia, and lung abscess. Aspergillosis was in the differential diagnosis, but at the lower end. No serological test was performed as a diagnostic workup for aspergillosis. The minimum emergent laboratory work-up did not indicate a mass of infectious disease source. Following surgery, Posaconazole, Amphotericin B, and other aggressive supportive care were given to the decedent, but the decedent was critically ill. Despite treatment, her condition continued to decline to a situation where death is imminent and palliative care to alleviate distressing symptoms are continued. All antifungal treatment was stopped after 2 days of treatment, as per family request. The decedent died in two weeks following surgery. The family requested only comfort care, and therefore, during the next 12 days, only comfort/palliative care was given. This autopsy was performed at our forensic medical autopsy office in Kansas upon family request and the autopsy was labeled as a "private case", The hospital or county coroner did not authorize an autopsy. The family wanted to know the diagnosis and cause of death. The autopsy examination was performed by an American board certified forensic pathologist and reviewed by another American board certified forensic pathologist. 
Autopsy of the decedent demonstrated disseminated invasive aspergillosis with multiple cavitating, necrotic, hemorrhagic, friable, soft, red and yellow-white lesions in both lungs (Figure 1), severe pericardial and pleural adhesions of the lungs, and hemorrhagic and cavitating soft masses in cerebrum (Figure 2) and cerebellum. Histologic examination of lungs revealed necrotizing pneumonia with sharply delineated rounded foci with hemorrhagic borders. Alveoli are filled with neutrophilic exudates with breakdown of the alveolar septa and formation of abscesses (Figure 3). In the brain there were multiple cavitating, hemorrhagic, friable, necrotic, soft yellow lesions. Microscopic sections demonstrated red blood cells and inflammatory cells in the involved area (Figure 4). GMS staining showed septate hyphae that branched dichotomously at acute angles, consistent with Aspergillus fungus (Figures 5 \& Figure 6). The fungal colonies were confined to the necrotic material and appeared as branching, slender, septate hyphae (Figures $5 \&$ Figure 6). There was no evidence of infection of the heart, pancreas, kidneys, liver, spleen, or adrenal glands.

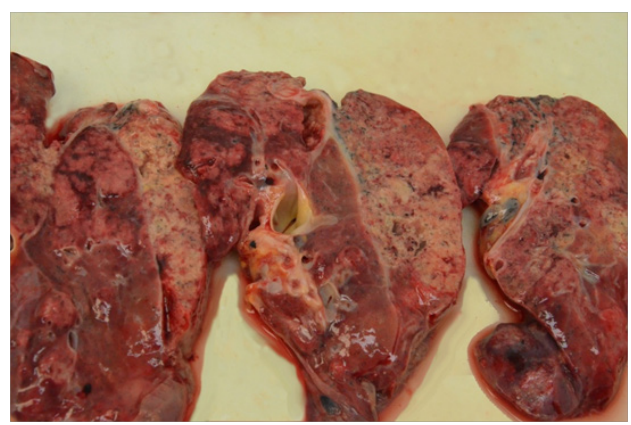

Figure I Gross view of lungs with necrotic, friable, soft, and yellow-white lesions.

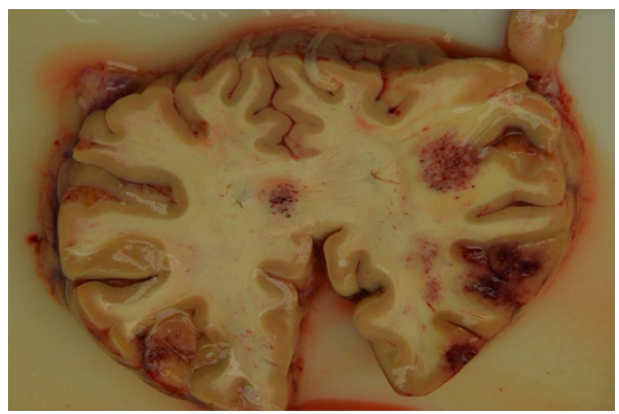

Figure 2 Coronal section of brain with necrotic, hemorrhagic, friable, soft, red and yellow-white lesions.

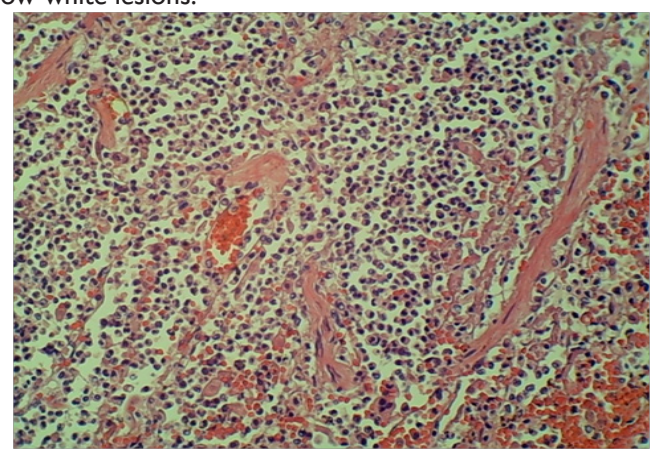

Figure $3 \mathrm{H}$ \& E, lung, 20x:Alveoli are with neutrophilic exudates with breakdown of the alveolar septa and formation of abscesses.

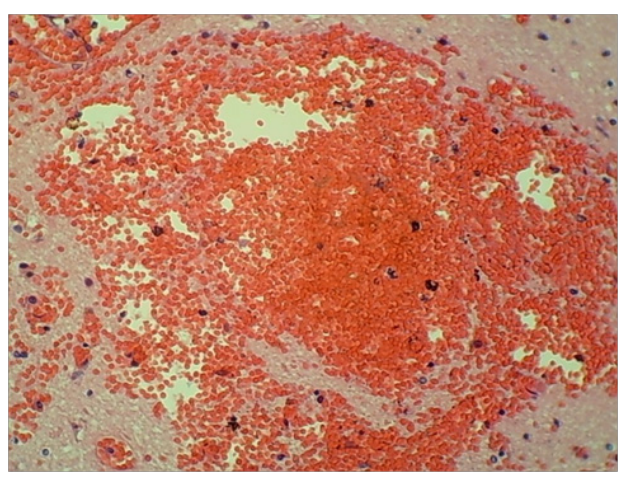

Figure $4 \mathrm{H}$ \& E 20xbrain:RBC and inflammatory cells.

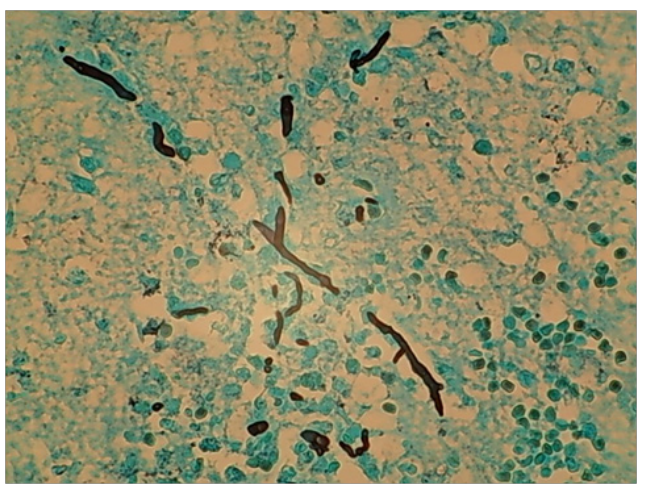

Figure 5 GMS stain, 40x, brain:Aspergillus species.

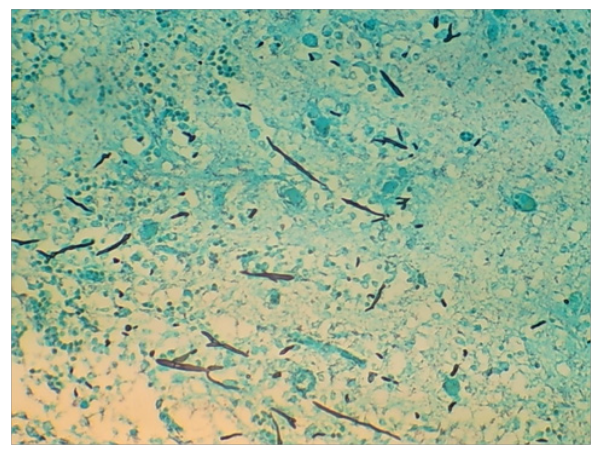

Figure 6 GMS stain 20x, brain:Aspergillus.

\section{Discussion}

Aspergillosis is a potentially life-threatening infection, caused by opportunistic fungi belonging to the genus Aspergillus. These fungi commonly exist in soil, on decaying plants, and in the atmosphere, especially in immuno suppressed patients. Though there are probably about 180species of Aspergillus, only a few of these causes human disease, especially A fumigatus, rarely A niger. Recently, Aspergillosis has become a significant healthcare problem for immunocompromised patients as relate to hematological malignancies, solid organ transplantation, AIDS, chemotherapy or steroid use. ${ }^{2}$ Aspergillosis infection is also reported when diabetes mellitus leads to immuno insufficiency in the host. ${ }^{3}$ Although the main entry of the mold into the human body generally occurs by inhalation of the conidia, an inoculation of conidia through damaged skin is not an infrequent 
way of gaining access to infection. The decedent had soft tissue and lung infection without a historically defined mechanism of introduction. Hematogenous dissemination is facilitated by several factors, especially the immuno suppression of the host and associated accompanying chronic diseases. The decedent had chronic obstructive lung disease and repeated bouts of pneumonia.

Diagnosis of invasive aspergillosis is often made late in the course of the disease due to the lack of reliable and feasible diagnostic techniques. Early diagnosis is critical in order to commence an appropriate treatment and avoid potentially deleterious outcomes. ${ }^{4}$ The decedent has disseminated aspergillosis precluding successful surgical cure though lobectomy might be helpful with recurrent hemoptysis. Recent literature review indicates Aspergillus organism became the most common pathogenic worldwide, still aspergillosis is often missed. There is increase in the number of aspergillosis in immunosuppressed patients. The average incidence of invasive aspergillosis at autopsy is $0.19 \% .^{5}$ It is increasingly being reported in AIDS patients (1-12\%) and also a common complication of chronic granulomatous disease of $25-40 \%{ }^{6}$ The most common symptoms of invasive aspergillosis are non-specific and include: fever, chest pain, cough, malaise, weight loss and dyspnea. Our case had these symptoms. However, approximately $41 \%$ of patients with invasive aspergillosis have no respiratory symptoms and corticosteroids treated patient frequently do not have elevated temperature. Serological test has been used to diagnose aspergillosis, but Invasive aspergillosis still remains a big clinical challenge and diagnosis relies largely on histopathological evidence of mycelial growth in tissue. Unfortunately, for many such cases where histology is needed in most cases and sometimes the diagnosis becomes available only at autopsy. ${ }^{8}$ Autopsy studies and articles on Aspergillus pneumonia or aspergillosis in forensic journals are relatively rare. Here we present a severe case of aspergillosis and the cause of death in this decedent was angioinvasive aspergillosis, involving lungs and brain. Aspergillosis was unexpected and unsuspected in this case. The decedent was suspected clinically as a case of either lung tumor, exacerbation of COPD or pneumonia. The clinicians, forensic pathologist and coroners should keep aspergillosis as one of the differential diagnosis.
A high index of suspicion, vigorous search for fungi by cultures and empirical treatment for highly suspicious cases remain the mainstay of management of these cases. Predisposing factors should be taken into account and if such are present empirical therapy started immediately, due to rapid progression of the disease and its severity.

\section{Acknowledgements}

None.

\section{Conflicts of interest}

The author declares no conflict of interest.

\section{References}

1. Aleksenko A, Gyasi RK. Disseminated Invasive Aspergillosis. Ghana Med J. 2006;40(2):69-72.

2. Winn W, Allen S, Janda W, et al. Koneman's Color Atlas and Textbook of Diagnostic Microbiology. 6th ed. Philadelphia: Lippincott Williams \& Wilkins; 2006. p. 1-30.

3. Norlinah MI, Ngow HA, Hamidon BB. Angioinvasive cerebral aspergillosis presenting as acute ischemic stroke in a patient with diabetes mellitus. Singapore Med J. 2007;48(1):e1-e4.

4. De Lone DR, Goldstein RA, Petermann G, et al. Disseminated aspergillosis involving the brain: distribution and imaging characteristics. AJNR Am J Neuroradiol. 1999;20(9):1597-1604.

5. Aideeswar P, Presed S, Deshande JR, et al. Invasive pulmonary aspergillosis: A study of 39 cases at autopsy. J Postgrad Med. 2004;50(1):21-26.

6. Latge JP. Aspergillus fumigatus and aspergillosis. Clin Microb Rev. 1999;12(2):310-350

7. Ribaud P, Chastang C, Latge JP, et al. Survival and prognostic factors of invasive aspergillosis after allopenic bone marrow transplantation. Clin Infect Dis. 1999;28(2):322-330.

8. Boon AP, O Brien D, Adams DH. 10 years review of invasive aspergillosis detected at necropsy. J Clin Path. 1991;44:452-454. 